\title{
ERP - SYSTEMS, LOGISTICS AND MECHATRONICS SYSTEMS FOR ENSURING THE SMOOTH CONSTRUCTION PROCESS
}

\author{
Alexey Grigoryevich Bulgakov ${ }^{1^{\star}}$ Georgii Evgenyevich Tokmakov² \\ ${ }^{1}$ Southwest State University, Kursk, Russia \\ ${ }^{2}$ South-Russsian State Polytechnic University, Novocherkassk, Russia
}

The article considers possible structures to integrate ERP systems at a building enterprise with low - end information and mechatronic systems - AMS (automated management systems) and MES which handle technological divisions as well as read necessary information. To achieve the information integrity of the construction process a multiagent control system of information and resource flows of industrial divisions together with a mathematical model of the operation is suggested.

Key words: ERP - system, MES - system, Logistic service, TIA technology, Intelligent agent, Mechatronic systems

\section{INTRODUCTION}

The main challenge in designing mechatronic systems (MS) is developing such MS configura-tions which main function is control manoeuvrability.

At present, this trend is connected with the realization of the decentralized control of technologi-cal divisions. In this case, the industrial system is thought to be a conductor of basic information and resource flows of the system (Figure 1), which means it should ensure a high control maneu-verability.

For the last 20-30 years, the integration of such production systems has reduced the production - related costs to an extent, which the present stage of science and technology development makes it possible. The intensification of specialization as an indispensable factor of the phe-nomenon in its turn implied an adequate development of cooperation and integration of manag-ing subjects.

Marketing goods with relatively short life spans accompanied by increasing consumer demands on the product quality has led to substantial changes of both goals and logistic service technol-ogy The conventional supply chain despite introducing logistics as functional management re-sponsible for material flow control and corresponding information and finance within the cycle of some companies could not ensure the level similar to the production field.

This is why the development of such logistic concept as Lean Production (LP) and the Just -in-time principle in 1990's was further shaped into the concept of Supply Chain Management (SCM) which is defined as the integration of key business processes starting with the final consumer and embracing all suppliers of goods, services and information thus adding value for consumers and other interested individuals.

\section{SUPPLY CHAIN MANAGEMENT, ERP-SYSTEMS, LOGISTIC}

When the term SCM was first introduced, it was used as synonym to "logistics" and "logistic management". In recent years, the meaning of the SCM category has changed and is now con-sidered a new business concept. Positive attitude to the SCM concept has resulted in reconsid-ering the definition of logistics as it is. According to the definition given by the Logistic Manage-ment Council (USA) in 1998 logistics is part of the process in supply chains wherein an effective and efficient flow of goods their supplies, service and related information is planned, imple-mented and controlled from the point of their originating to the point of their consumption in order to meet the consumer demands [01].

So the supply chain management tends to be a more global category than logistics. It is the network, which prevails as an organizational structure rather than the hierarchy. The network of small systems is far more sensitive to market changes than large bureaucratic structures. Specialists no longer argue about key business processes of SCM related to different levels of the control system which interaction is realized in the network structure of the industrial communica-tion system.

The components of such systems implementing TIA technology $[02,03]$ in the SIMANTIC NET, shown in Figure 2, serve as the fundamentals to provide industrial communication in case of large scale construction projects, within the frame of a construction object, in the technological pro-cess. There have been suggested ways to solve control problems, which make the most of all the advantages of the Ethernet and imply vertical data communication from the field level to the corporate management level with a wide use of information technologies. 


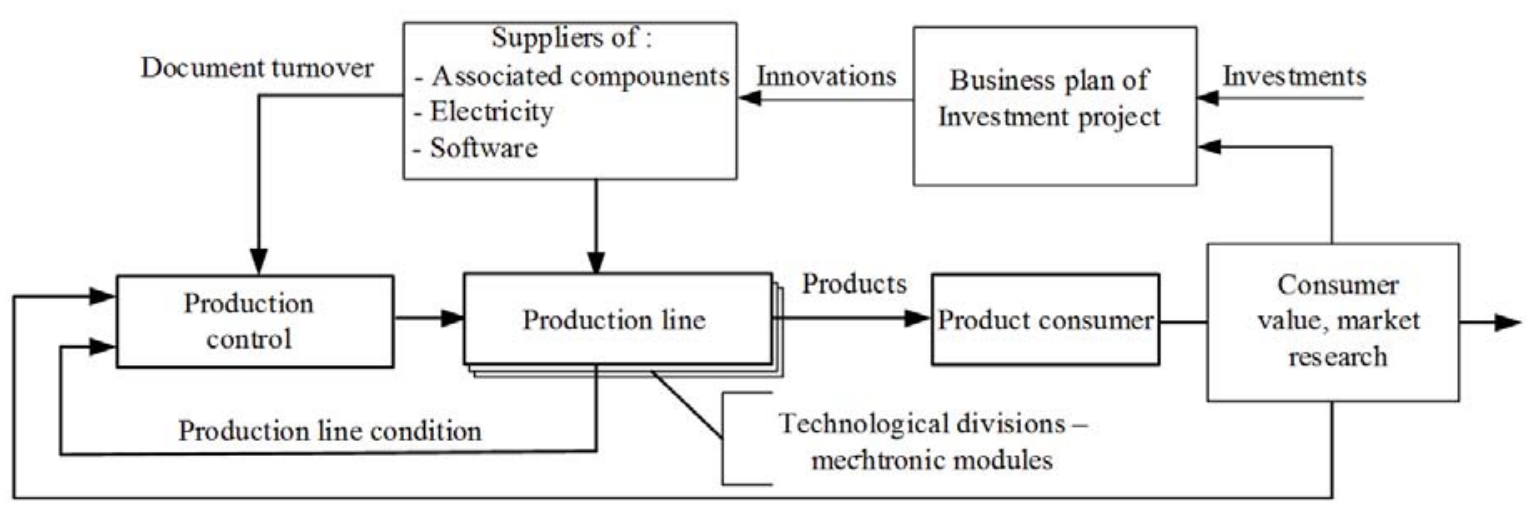

Figure 1: Basic information and resource flows of production system



Figure 2: Network communication in TIA-technology

The development of information integrity is progressing from connecting separate automated areas to programmed cooperation of all offices, design departments and technological divisions. With soft web-technologies, such integration can be realized through online channels of several enterprises, suppliers and even consumers. The ways of integration of multiple functions at the plant as well as outside it are as varied as the ways of local automation and construction pro-duction control. The advantages of such programmed integration being advertised or as count-less as the quantity of different exact customer conditions.

The research made by many authors [04-07] and consumer data speak of the fact that the effec-tive interaction of different levels increases the production growth rate without plant refurbishing by $10-25 \%$. The set of the function performed can be implemented by way of specific intelligent modules or increasingly as off-the-shelf software packages of one or several suppliers. I due time software manufactures divided the market and found their own areas corresponding to dif-ferent plant levels: systems of regulation execution production systems cooperate management systems as well as CAD and office systems. However, within the time the borders between them are beginning to smooth over. The customers' need of greater availability and cheaper ways of connecting systems to make information obtained from different divisions compatible appeared.

\section{INTELLIGENT AGENT CONCEPT}

The influence of the Internet on the efficiency of business activity has resulted in making selling goods with decision making concerning planning projects, their implementation, material shipping and handling all the supply chain through the "Portals of Intelligent Logistics". The key to understanding the "Intelligent Logistics" is the "Intelligent Agent Concept".

The basic feature of the second-generation multiagent logistics is replacing the bar code with electronic markers, each of them containing an agent. 
This kind of commodity coding will enable to develop mechatronic systems for monitoring and material handling through the delivery process from the supplier to the construction site. These mechatronic systems based on the multiagent structure to control business processes within the ERP system will incorporate the following elements:

- Intelligent distribution of goods on the shelves (in warehouses).

- Intelligent robots capable of searching goods according to the data read from the intelligent codes, unlike conventional robots, which can just deliver a commodity to a definite point.

- Intelligent transport systems (lorries, stand-alone means of transport, conveyors) capable of determining the kind of goods contained in them and transmitting this information further along the system if necessary.

- Intelligent systems designed to control the environment of storing goods and which a capable of detecting the proper conditions for storing each of the commodities.

- Intelligent commodity sorting systems that can sort out commodities in accordance with the data recorded in their codes.

In order to control information and resource flows of mechtronic production system considered above (Figure 1) a team of five agents can be organized responsible for planning, maintenance, operation, safety and administration, each of them being a miniature system with knowledge base which functioning can be represented by three stages: setting a goal, receiving results, evaluating operation effectiveness.

Each agent performs one operation in S0-system, that is the production line making this opera-tion and that is why the execution of the operation can be thought to be a process of changing its states in the multitude $(Z)$ of all possible states of SO-system. In this case an intelligent agent of S0-system is a mathematical model of the operation $(\Psi)$ designed to solve the problem of quantitative evaluation of effectiveness (W) as the criterion of the rate of the correspondence of an actual(predicted) result $(Y)$ of the operation to the result desired $\left(Y^{d}\right)$ or the goal achievement rate $\left(A_{0}\right)$ of the operation, expressed by the result desired $\left(Y^{d}\right)$.

Quantitative and generally vector numerical values $(\mathrm{W}(\mathrm{u}))$ are obtained for all strategies $(\mathrm{u})$ from the multitude of possible strategies considered. Each of these values $(\mathrm{W}(\mathrm{u}))$ characterizes a level of effectiveness(goal achievement rate) of the operation in use of the strategy given $(u \in U)$.

In general the problem of evaluation of operation effectiveness can be represented by a formal equation of the following kind:

$$
\begin{aligned}
& W(u)=M\left[\rho\left(Y(u), Y^{d}\right)\right] \\
& \psi:\left\{Y \mid H: U \times \Lambda^{\theta} \rightarrow Y(G)\right\}^{\theta \rightarrow W}
\end{aligned}
$$

where $\Lambda$ - multitude of definite and indefinite factors forming the environment of the operation; $Y^{d}$ - operation result desired; $Y$ - operation result that is the vector of characteristics of the out-come ( $g \in G$ ) of the operations significant for achieving the goal $\left(A_{0}\right)$ of the operation, expressed by the result desired $\left(Y^{d}\right) ; M$ - a sign of mathematical expectation; $\rho-$ a function of correspond-ence; $\mathrm{H}$ - a model of the operation result enabling to calculate the values $(\mathrm{Y}(\mathrm{u}))$ of the operation result $(\mathrm{Y})$ for each strategy $(u \in U)$

$\Psi$ in (2) is the reflection of the multitude (U) of possible strategies into the multitude of values of effectiveness indicator (W) with consideration of (1) and is usually given in the form of a definite mathematical model of the operation.

In the multiagent control system of information and resource by $(1,2)$ can be used to create a structure of programmed genes suitable for all five agents. Such structure shown in Figure 3 enable us to evaluate the effectiveness of operations, for example, planning and maintenance. The agent of planning has a goal to reduce the cost of products $\left(\mathrm{A}_{0}\right)$ and to determine a rational volume and types of orders for manufacturing this or that product on the basis of the business plan $(\Lambda)$ and the results of market research. To be able to choose the best marketing steps ( $U^{*}$ - strategies) the agent "holds the negotiations" with suppliers in the form of an auction using submodels $\left(P_{u}, P_{\Lambda}\right)$ which are models of preferences $(P)$. Similarly on the basis of submodels of preferences $\left(P_{G}\right.$ $\left.P_{Y}\right)$ and with consideration of the data $\left(\theta_{H}\right)$ of available means to build the models $(H)$ the characteristics $(Y)$ of the outcome $(G)$ are chosen, the kind of correspondence $(H: U \times \wedge \rightarrow Y(G))$ is set and the value of the result desired $\left(Y^{d}\right)$ is estimated.

Then based on the data $\left(Y, Y^{d}\right)$ and preferences $\left(P_{w}\right)$ concerning the type of the effectiveness indicator one of the possible kinds of the parameters $\left(\rho\left(Y, Y^{d}\right)\right)$ is set and the model "result-indicator" is formed. At the same time the criterion $(K)$ based on the data and submodel $\left(P_{K}\right)$ can offer a criterion in the form of the decisive rule. Based on the consideration about the goal achievement rate of the operation the best option from the multitude $\left(U^{*} \in U\right)$ is picked up or some elements of the model of the problem situation are returned and corrected.

Thus in the process of receiving results the correlation (2) is obtained while analyzing the results leads to the following correlation:

$$
P{ }^{\theta} \rightarrow K: U \quad W \rightarrow U^{*}
$$

The goal of the agent of maintenance is to minimize time and energy consumption necessary to produce a product. A great deal of factors $(\Lambda)$ form the agent knowledge base of the technical state of the line, which possible configurations $(U)$ make it possible to estimate its technical ca-pabilities considering the models of preferences for the best marketing steps of the agent of planning. 


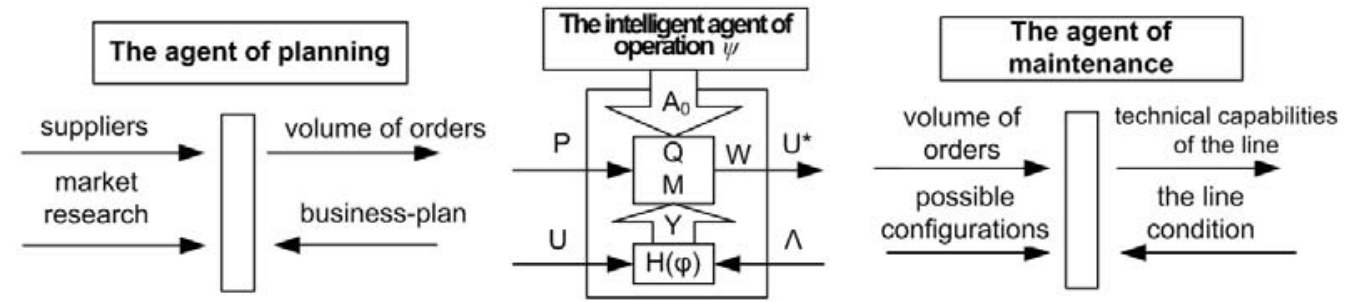

Figure 3: The structure of the intelligent agent for the operation

The parameters $(Q, M)$ reflect the multitude of the operation results $(Y)$ into the multitude of the effectiveness indicator $(\mathrm{W})$. Under this circumstance the multitude $(\mathrm{Y})$ together with the parame-ter of the correspondence $(Q$ : $\left.Y \times Y^{d}\right)$ is reflected into the multitude of the function of the corre-spondence $(\rho)$, while the operator for averaging out transform the multitude of the values of the function of the correspondence $(\rho)$ into the multitude of the values of the effectiveness indicator $(W)$ i.e. $Q \circ M: Y \rightarrow W$. The superposition of the parameters $Q \circ M$ determines the correlation of given effectiveness indicator to the operation goal $\left(A_{0}\right)$ expressed by the result desired $\left(Y^{d}\right)$. A certain type of the parameter depends on the behavior of numerous factors and relations be-tween them which must be considered while evaluating effectiveness.

When certain properties of the operation are not given the agent has a possibility to determine the trajectories of functioning of the system $\left(Z_{T}, Y_{T}\right)$, whereas the data of input effects $(U \times \Lambda)$ can be used to predict both the operation result $(\mathrm{Y})$ and the state of the $\mathrm{S} 0$-system. In this case the parameters $(\varphi, \mathrm{H})$ called transform and output parameters correspondingly can be introduced and recorded in the following:

$$
\begin{aligned}
& \varphi: Z \times U \times T \times \Lambda \rightarrow Z \\
& H: Z \times U \times T \times \Lambda \rightarrow Y(G)
\end{aligned}
$$

Each point in the trajectory $\left(Z_{T}\right)$ of the states of the $\mathrm{S}_{0}$-systems and in the trajectory $\left(\mathrm{Y}_{\mathrm{T}}\right)$ of the operation results found with the help of the parameters (4) and (5), characterizes the state of the system $\left(Z_{t}\right)$ and the value of the operation result $\left(Y_{t}\right)$ at a definite moment of the time.

\section{CONCLUSIONS}

Thus, now approach to the organization of construction production has to be not just integrated approach, but multi disciplinary. In organization of construction process automated management systems is required not only for control of the construction equipment, but also in general for the organization for ensuring the smooth construction process.

\section{REFERENCES}

1. G. Rzevski. On Behaviour and Architectures of Autonomous Intelligent Agents: An Engineer-ing Perspective, Proceedings First International Round-Table on Abstract Intelligent Agents, ENEA, Rome, 1993.

2. TIA - technology // Siemens electronic information resource URL: http://www.automation.siemens.com/ $\mathrm{mcms} /$ topics/en/tia/Pages/default.aspx?stc=wwiia303256 (date of reference: 04.03.2010).

3. Mark T. Hoske. How to connect office, design and control systems, CONTROL ENGINEER-ING //The world of computer automation; On-line., October 1997. URL: http://www.mka.ru/?p=40188 (date of reference: 09.09.2010) Reliability and effectiveness in technology: Reference book./editing board: Aduevsky D. - Moscow.: Machinebuilding, 1988.

4. K.K. Shestopalov. Hoisting-and-transport, building and road machines and equipment. M.: Academy, 2009. P. 67-72.

5. S.W. Hughes, D.D. Tippett, W.K. Thomas. Measuring project success in the construction industry // EMJ - Engineering Management Journal. 2004. 16(3). P. 31-37.

6. Min-Yuan Cheng, Hsing-Chih Tsai, Erick Sudjono. Evolutionary fuzzy hybrid neural network for construction industry. Automation in Construction 21 (2012) S. 46-51.

7. T.M. Cheng, C.W. Feng, M.Y. Hsu. An integrated modeling mechanism foroptimizing the simulation model of construction operation, Autom. Constr. 15 (2006) 327-340.

Paper submitted: 26.07.2017.

Paper accepted: 02.09.2017.

This is an open access article distributed under the CC BY-NC-ND 4.0 terms and conditions. 